\section{Response to "Data limitations may affect conclusions in study of vaginal delivery at midpelvic station"}

We thank Dr. Wood ${ }^{1}$ for his interest in our article ${ }^{2}$ and his comments. Respectfully, we disagree with several of his assertions, as they are unfounded or incorrect.

Our main analysis was restricted to deliveries that occurred after a prolonged second stage of labour, to ensure an appropriate reference group (i.e., cesarean deliveries that occurred in the second stage of labour). Although the flow chart in Figure $1^{2}$ indicates that 226626 women were excluded because of an "uncertain indication for operative delivery," this group of excluded women overlapped markedly with the 290328 women who were excluded because "no labour [was] recorded" (i.e., elective cesarean deliveries that were not relevant to our study). Thus, only the remaining 13634 women had operative vaginal or cesarean deliveries with labour with an uncertain indication for operative delivery. The latter represent $7.3 \%$ of all operative vaginal or cesarean deliveries in labour.

The statement that the study was restricted to women with dystocia who had a prolonged second stage is incorrect. Our paper quantified the effects of operative vaginal delivery at midpelvic station among women with dystocia, with fetal distress, without a prolonged second stage, and with a prolonged second stage of labour.

Dr. Wood asserts that the validation studies cited in our paper evaluated maternal outcomes but not neonatal outcomes. In fact, the first paper cited ${ }^{3}$ evaluated neonatal diagnoses coded using International Classification of Diseases 10th Revision (ICD-10-CA) codes. Examples of neonatal diagnoses validated in the cited study ${ }^{3}$ included intraventricular hemorrhage (sensitivity $89 \%$, 95\% confidence interval $[\mathrm{Cl}]$ 52\%-99.7\%; specificity $100 \%$, 95\% Cl 99.9\%-100\%), fracture of the clavicle (sensitivity $91.7 \%$,
95\% Cl 62\%-99.8\%; specificity 100\%, 95\% Cl 99.3\%-100\%), severe respiratory distress syndrome (sensitivity $100.0 \%$, 95\% Cl 95.5\%-100\%; specificity 99.6\%, 95\% Cl 99.4\%-99.8\%), and respiratory distress syndrome (sensitivity 50.9\%, 95\% Cl 43.1\%-58.6\%; specificity 99.8 , 95\% Cl 99.7\%-99.9\%).

Diagnostic suspicion bias is a potential threat in many nonexperimental studies. Such a bias is typically addressed by focusing on severe outcomes (because less serious outcomes are more likely to be differentially identified, whereas severe outcomes are more likely to result in complete and hence nondifferential ascertainment). Thus, the primary outcome in our study included only severe birth trauma; viz., intracranial laceration and hemorrhage, skull fracture, severe injury to the central or peripheral nervous systems, long bone injury, subaponeurotic hemorrhage and injury to the liver or spleen. Scalp lacerations and facial nerve palsy were not included in severe birth trauma, although they were included in our secondary outcomes of any birth trauma. However, our findings do not provide evidence of a diagnostic suspicion bias: for example, the adjusted odds ratio (OR) quantifying the effect of attempted midpelvic forceps on severe birth trauma in women with fetal distress and a prolonged second stage was 10.4 (95\% Cl 4.84-22.5), which was significantly higher than the same adjusted OR for any birth trauma $(3.26,95 \% \mathrm{Cl} 2.65-$ 4.02; Table 3). ${ }^{2}$

The study that Dr. Wood cites (Whitby and colleagues ${ }^{4}$ ) does not support his contention that asymptomatic subdural hemorrhage in newborns is common across all modes of delivery. This small study reported nine cases of asymptomatic subdural hemorrhage among 111 newborn infants. Rates of asymptomatic subdural hemorrhage were $16 \%$ following operative vaginal delivery, $6.1 \%$ following spontaneous vaginal delivery and $0 \%$ following cesarean delivery. ${ }^{4}$

Because intracranial hemorrhage is an uncommon outcome, our study's ability to detect significant associations between mode of delivery and intracranial hemorrhage was limited by a lack of statistical power. For this reason, we created a composite severe birth trauma outcome in an attempt to provide a meaningful estimate of the effect of midpelvic operative vaginal delivery on severe birth trauma. We conclude that severe birth trauma is higher following attempted midpelvic operative vaginal delivery based on the 4- to 10-fold higher rates of severe birth trauma following attempted midpelvic forceps or midpelvic vacuum delivery compared with cesarean delivery (numbers needed to treat ranging from 68 to 168).

\section{Giulia M. Muraca MPH}

School of Population and Public Health, Department of Obstetrics \& Gynaecology, The University of British Columbia, Vancouver, BC

\section{Amanda Skoll MD}

Associate Professor, School of Population and Public Health, Department of Obstetrics \& Gynaecology, The University of British Columbia, Vancouver, BC

\section{Sarka Lisonkova MD PhD}

Associate Professor, School of Population and Public Health, Department of Obstetrics \& Gynaecology, The University of British Columbia, Vancouver, BC

\section{Yasser Sabr MD MHSC}

Graduate Student, Department of Obstetrics \& Gynaecology, The University of British Columbia, Vancouver, BC; Assistant Professor, Department of Obstetrics and Gynaecology, King Saud University, Riyadh, Saudi Arabia

\section{Rollin Brant PhD}

Professor, Department of Statistics, The University of British Columbia, Vancouver, BC 
Geoffrey W. Cundiff MD

Head and Professor, Department of Obstetrics \& Gynaecology,

The University of British Columbia, vancouver, BC

\section{K.S. Joseph MD PhD}

Professor, School of Population and

Public Health, Department of

Obstetrics \& Gynaecology,

The University of British Columbia, Vancouver, BC

Cite as: CMAJ 2017 October 30;189:

E1344-5. doi: 10.1503/cmaj.733350

\section{References}

1. Wood S. Data limitations may affect conclusions in study of vaginal delivery at midpelvic station [letter]. CMAJ 2017;189:E1343.

2. Muraca GM, Skoll A, Lisonkova S, et al. Perinatal and maternal morbidity and mortality after attempted operative vaginal delivery at midpelvic station. CMAJ 2017;189:E764-72.

3. Joseph KS, Fahey J. Canadian Perinatal Surveillance System. Validation of perinatal data in the Discharge Abstract Database of the Canadian Institute for Health Information. Chronic Dis Can 2009;29:96-100.

4. Whitby EH, Griffiths PD, Rutter S, et al. Frequency and natural history of subdural haemorrhages in babies and relation to obstetric factors. Lancet 2004;363:846-51.

Competing interests: None declared. 